\title{
Traitements de dénaturation appliqués à la $\beta$-lactoglobuline avant hydrolyse trypsique
}

\author{
C lung *, D Pâquet, G Linden \\ Laboratoire de biochimie appliquée, associé à I'INRA, Université de Nancy l, Faculté des Sciences, \\ $B P 239,54506$ Vandœuvre-lès-Nancy cedex, France
}

(Reçu le 10 août 1989; accepté le 11 février 1991)

\begin{abstract}
Résumé - La $\beta$-lactoglobuline est isolée en 2 étapes à partir de lactosérum acide par chromatographie d'échange d'ions en "batch" (DEAE-Sephacel) suivie de chromatographie de filtration sur gel (Sephadex G-100).

On réalise soit un traitement thermique à $90^{\circ} \mathrm{C}$ en milieu acide dilué, soit une coupure des ponts disulfures en milieu dénaturant. La solubilité au pHi est de $99,5 \%$ pour la $\beta$-lactoglobuline native, $38 \%$ pour le substrat chauffé en milieu acide et $5 \%$ pour la $\beta$-lactoglobuline réduite. Ces différents substrats sont alors soumis à un traitement enzymatique par la trypsine à $37^{\circ} \mathrm{C}$ et $\mathrm{pH} 7,5$. Les produits obtenus sont caractérisés par la mesure des liaisons peptidiques rompues (méthode au TNBS) et analyse électrophorétique en milieu dissociant (évaluation du poids moléculaire).

L'hydrolyse trypsique du substrat dans l'état natif conduit à l'apparition de peptides de petites tailles. Le chauffage en milieu acide dilué modifie le substrat de départ et de nombreux polypeptides apparaissent; la trypsine hydrolyse très rapidement ces produits. La coupure des ponts disulfures induit une hydrolyse de la $\beta$-lactoglobuline par la trypsine en peptides de taille importante qui sont à leur tour hydrolysés.

Ces observations viennent souligner l'importance des pré-traitements appliqués à un substrat et l'intérêt que peut présenter la combinaison de traitements enzymatiques, thermiques et chimiques.
\end{abstract}

$\beta$-lactoglobuline / traitement thermique en milieu acide / réduction des ponts disulfures / hydrolyse trypsique

Summary - The effects of denaturation treatments on tryptic hydrolysis of $\beta$-lactoglobulin. The extraction of $\beta$-lactoglobulin from acid whey was achieved by ion exchange chromatography in batch (DEAE-Sephacel) followed by gel filtration chromatography (Sephadex G-100). The protein was denatured either by thermal treatment at $90^{\circ} \mathrm{C}$ in diluted acid media or by cleavage of disulfide bonds. Solubility at the isoelectric $\mathrm{pH}$ of $99.5 \%$ was obtained for the native $\beta$-lactoglobulin, of $38 \%$ for the thermal treated substrate and of $5 \%$ for the reduced $\beta$-lactoglobulin. These different substrates were incubated with trypsin at $37{ }^{\circ} \mathrm{C}, \mathrm{pH} 7.5$ with various $\mathrm{E} / \mathrm{S}$ ratios. Progress of hydrolysis was determined by measuring the number of peptide bonds cleaved by the TNBS method and by electrophoresis on SDS-polyacrylamide gel. With native $\beta$-lactoglobulin, the degree of hydrolysis exceeding $10 \%$ at $24 \mathrm{~h}$ could be obtained only at high E/S ratio (1/50 and 1/25): low molecular weight peptides were produced and could not be revealed by electrophoresis.

\footnotetext{
*Adresse actuelle : La Prospérité Fermière, 51-53, avenue Fernand Lobbedez, BP 946, 62000 Arras Cedex, France
} 
Partial denaturation by thermal treatment at $90^{\circ} \mathrm{C}$ in media of low acid concentration $(0.02 \mathrm{M} \mathrm{HCl})$ induced an alteration: a set of polypeptides were identified by electrophoresis. This product seemed to be susceptible to tryptic hydrolysis. The reduction of disulfide bonds resulted in the appearance by tryptic hydrolysis of intermediate peptides detected by electrophoresis which were then gradually transformed into other final products. These results show the influence of pretreatment on the proteol$y$ sis of $\beta$-lactoglobulin and indicate the interest of combined enzymatic, thermal and chemical treatments.

\section{$\beta$-lactoglobulin / thermal treatment / disulfide bond reduction / tryptic hydrolysis}

\section{INTRODUCTION}

La $\beta$-lactoglobuline est la protéine majeure du lactosérum (environ $50 \%$ des protéines lactosériques, soit 2 à $4 \mathrm{~g} / \mathrm{l}$ de lait). Elle joue un rôle important dans tous les produits laitiers et les aliments dans lesquels les protéines de lactosérum sont ajoutées comme ingrédients. Les études sur les propriétés de la $\beta$-lactoglobuline ont trait à ses propriétés nutritionnelles et en particulier à la disponibilité de ses acides aminés (Miranda et Pélissier, 1983; Yvon et al, 1984), aux réactions d'allergénicité (Jakobsson et al, 1985; Jost, 1988) ou encore à ses propriétés fonctionnelles, en particulier ses propriétés de surface (Waniska et Kinsella, 1985; Shimizu et al, 1985).

La $\beta$-lactoglobuline possède une structure globulaire maintenue par 2 ponts disulfures. Sa structure tridimensionnelle a été déterminée récemment par Sawyer et al (1985) et Papiz et al (1986).

Dalgalarrondo et al (1990) identifient la partie $\mathrm{N}$-terminale comme particulièrement susceptible à l'hydrolyse trypsique à pH 8 . Mohan Reddy et al (1988) relient les changements conformationnels obtenus par des traitements thermiques en milieu neutre à 80 et $90^{\circ} \mathrm{C}$ ou la coupure des liaisons disulfures à une diminution de la résistance de la $\beta$-lactoglobuline aux digestions pepsiques et chymotrypsiques. La S- carboxyméthylation en présence de $\beta$ mercaptoéthanol - réduction des ponts disulfures - augmente la susceptibilité de la protéine à l'hydrolyse par la pepsine, la trypsine ou la chymotrypsine (Otani, 1981). Ainsi, la résistance à la protéolyse de la $\beta$ lactoglobuline est attribuée à la structure spatiale compacte de la protéine sous sa forme native.

Nous nous proposons ici d'étudier l'influence de traitements dénaturants sur la dégradation enzymatique de la $\beta$ lactoglobuline par la trypsine.

\section{MATÉRIELS ET MÉTHODES}

Les gels DEAE-Sephacel et Sephadex G-100 proviennent de Pharmacia Fine Chemicals (Uppsala, Suède). Tous les réactifs sont de grade analytique et fournis par Sigma Chemical Co (St Louis, MO).

\section{Préparation de la $\beta$-lactoglobuline}

Du lait cru de petit mélange est écrémé par centrifugation $(2000 \mathrm{~g}, 30 \mathrm{~min})$ et acidifié à pH 4,6 par addition d'HCl concentré. Le lactosérum est récupéré par centrifugation $(2000 \mathrm{~g}, 30 \mathrm{~min})$ et filtration sur papier. La méthode d'obtention de la $\beta$-lactoglobuline à partir de ce lactosérum est celle décrite par lung et al (1987). Un fractionnement en ubatch" sur échangeur d'anions (DEAE-Sephacel) permet d'obtenir une fraction très fortement enrichie en $\beta$-lactoglobuline; une 
étape supplémentaire de filtration sur gel (Sephadex G-100) conduit à une protéine de degré de pureté supérieur à $99 \%$.

\section{Traitements de dénaturation}

La réduction des ponts disulfures est réalisée selon la méthode de Konisberg (1972) en milieu urée et présence de dithiothréitol puis blocage de la réaction à l'aide d'iodoacétamide. Le traitement thermique en milieu acide est réalisé sur une solution de $\beta$-lactoglobuline à $0,5 \%$ en milieu $\mathrm{HCl} 0,02 \mathrm{M} \mathrm{pH} \mathrm{2,5} \mathrm{à} 90^{\circ} \mathrm{C}$ pendant $1 \mathrm{~h}$. La solution est dialysée contre un tampon phosphate de sodium $0,2 \mathrm{M} \mathrm{pH} \mathrm{7,5.}$

\section{Mesure de la solubilité protéique}

Le $\mathrm{pH}$ de la solution protéique est amené à $\mathrm{pH}$ 5,1 (point isoélectrique de la $\beta$-lactoglobuline) à l'aide d' $\mathrm{HCl} 0,1 \mathrm{~N}$ et une centrifugation à $5000 \mathrm{~g}$ $30 \mathrm{~min}$ est appliquée. Le dosage des protéines est réalisé sur la solution initiale et sur le surnageant par la méthode de Lowry et al (1951).

\section{Hydrolyse trypsique}

La $\beta$-lactoglobuline en solution à $0,5 \%$ ou $1 \%$ dans le tampon phosphate de sodium $0,2 \mathrm{M} \mathrm{pH}$ 7,5 est équilibrée en température à $37^{\circ} \mathrm{C}$. La trypsine type XIII traitée TPCK (14500 U BAEE/ $\mathrm{mg}$ ) dissoute dans ce même tampon est ajoutée dans un rapport $E / S(P / P)$ de $1 / 200,1 / 100,1 / 50$ ou 1/25. Des aliquotes sont prélevées au cours du temps et inactivées à l'aide de l'inhibiteur trypsique de soja type I.S ajouté dans un rapport $E / I(P / P)$ de $1 / 2$. Les groupements $-\mathrm{NH}_{2}$ apparus sont dosés à l'aide de l'acide 2,4,6trinitrobenzène sulfonique (TNBS) selon la méthode citée par Church et al (1985) : sous des conditions alcalines, la réaction du TNBS avec les amines primaires donne naissance à un chromophore; cette réaction est stoppée par l'abaissement du $\mathrm{pH}$ et le dosage spectrométrique est réalisé à $340 \mathrm{~nm}$. Le degré d'hydrolyse (DH) représente le nombre de liaisons peptidiques coupées par rapport au nombre de liaisons peptidiques totales et sera exprimé en pourcentage.

\section{Electrophorèse en gel de polyacrylamide en présence de SDS}

La méthode de Laemmli et Favre (1973) est utilisée avec un gel de séparation à $15 \%$ d'acrylamide et un rapport acrylamide/bis-acrylamide de $37 / 1$ en tampon Tris/ $\mathrm{HCl} 0,38 \mathrm{M} \mathrm{pH} 8,8$ et un gel de concentration à $4,8 \%$ d'acrylamide et un rapport acrylamide/bis-acrylamide de $37 / 1$ en tampon Tris/ $\mathrm{HCl} 0,125 \mathrm{M} \mathrm{pH} \mathrm{6,8.}$

\section{RÉSULTATS ET DISCUSSION}

\section{$\beta$-lactoglobuline native}

La solutilité protéique à $\mathrm{pH} 5,1$ est de $99,5 \%$ (tableau I) et traduit donc le respect de la structure native de la $\beta$-lactoglobuline lors de la préparation. En effet, les protéines globulaires de lactosérum se caractérisent par une solubilité élevée sur toute la gamme de $\mathrm{pH}$ et une dénaturation entraîne une insolubilisation, observable en premier lieu dans la zone du $\mathrm{pHi}$ (Cheftel et al, 1985).

L'hydrolyse trypsique conduit à des degrés d'hydrolyse de 5 à $14 \%$ après $24 \mathrm{~h}$ selon le rapport E/S utilisé (1/200 à 1/25) (fig 1).

L'analyse électrophorétique (fig 2) traduit la diminution de la $\beta$-lactoglobuline au

Tableau I. Taux de solubilité au pHi de la $\beta$ lactoglobuline.

Solubility index at pHi of $\beta$-lactoglobulin.

Taux de solubilité (\%)

$\beta$-lactoglobuline native

99,5

$\beta$-lactoglobuline chauffée

en milieu acide

38,0

$\beta$-lactoglobuline réduite 


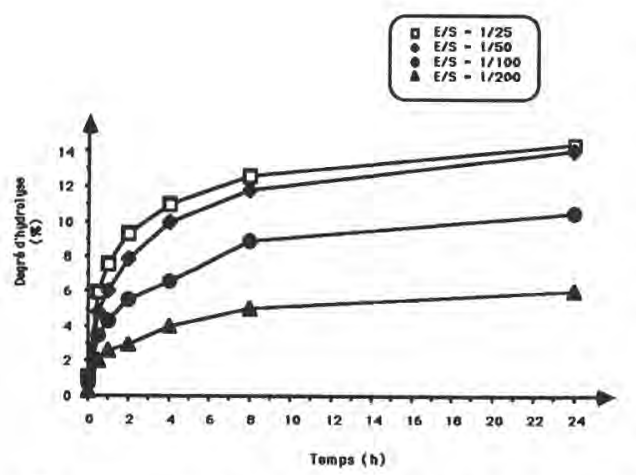

Fig 1. Degré d'hydrolyse en fonction du temps de la $\beta$-lactoglobuline par la trypsine. Concentration en $\beta$-lactoglobuline: $0,5 \%$; rapports enzyme/substrat $(p / p): 1 / 25,1 / 50,1 / 100,1 / 200$;

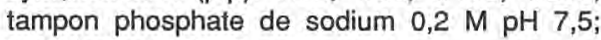
température : $37^{\circ} \mathrm{C}$.

Time course of tryptic digestion of $\beta$ lactoglobulin, $\beta$-lactoglobulin concentration: $0,5 \%$; E/S ratio $(w / w): 1 / 25,1 / 50,1 / 100,1 / 200$; Na phosphate buffer $0,2 \mathrm{M} \mathrm{pH} 7.5$; temperature: $37^{\circ} \mathrm{C}$.

cours de l'hydrolyse qui ne s'accompagne pas d'une apparition de produits intermédiaires de taille suffisante pour la résolution du gel (supérieure à $3000 \mathrm{Da}$ ). Les bandes au temps zéro correspondent à la trypsine et l'inhibiteur trypsique.

Les degrés d'hydrolyse atteints sont supérieurs à celui du calcul théorique pour l'hydrolyse totale par la trypsine qui s'élève à $12 \%$. Le rapport E/S très élevé (1/25) provoque des coupures non spécifiques au sein de la molécule de $\beta$ lactoglobuline: il en résulte au temps $4 \mathrm{~h}$ une présence résiduelle de $\beta$ lactoglobuline pour un degré d'hydrolyse de l'ordre de $11 \%$.

\section{$\beta$-lactoglobuline chauffée en milieu acide}

Le chauffage à $90^{\circ} \mathrm{C}$ en milieu $\mathrm{HCl} 0,02 \mathrm{M}$ $\mathrm{pH} 2,5$ pendant $1 \mathrm{~h}$ de la solution de $\beta$ lactoglobuline à $0,5 \%$ conduit à l'obtention d'une solution limpide; cette limpidité est conservée après dialyse contre le tampon $\mathrm{pH} 7,5$. Le taux de dénaturation apprécié par la perte de solubilité à pH 5,1 s'élève à $62 \%$ (tableau I). Selon Harwalkar (1980), cela correspond à 2 espèces moléculaires :

- une forme dénaturée, insoluble au $\mathrm{pHi}$ de la $\beta$-lactoglobuline;

- une forme "native" ou "quasi-native", toujours soluble au $\mathrm{pHi}$.

L'hydrolyse trypsique de ce produit est suivie pendant $2 \mathrm{~h}$ avec un rapport E/S de $1 / 25$ comparativement à la $\beta$-lactoglobuline native : le degré d'hydrolyse atteint $7,5 \%$ après 30 min (supérieur de 3,5\% par rapport au témoin) et $11 \%$ après $2 \mathrm{~h}$ (supérieur de $2 \%$ par rapport au témoin).

L'électrophorèse (fig 3) permet d'observer des changements importants de la $\beta$ lactoglobuline après chauffage en milieu acide : de nombreuses bandes dont les principales ont des poids moléculaires de 17000 ( $\beta C 1), 13000$ ( $\beta C 2), 9400$ ( $\beta C 3$ ), $6000(\beta C 4)$ et $3500(\beta C 5)$ sont identifiées. Ces produits sont très rapidement hydrolysés (disparition après $30 \mathrm{~min}$ ), ce qui correspond à la progression rapide du degré d'hydrolyse. $\mathrm{La}$ bande correspondant au poids moléculaire de la $\beta$-lactoglobuline est hydrolysée plus progressivement et reste décelable après $6 \mathrm{~h}$ d'hydrolyse.

Le traitement de chauffage en milieu acide dans les mêmes conditions que Harwalkar (1980) conduit à l'apparition de nombreux produits de dégradation mis en 


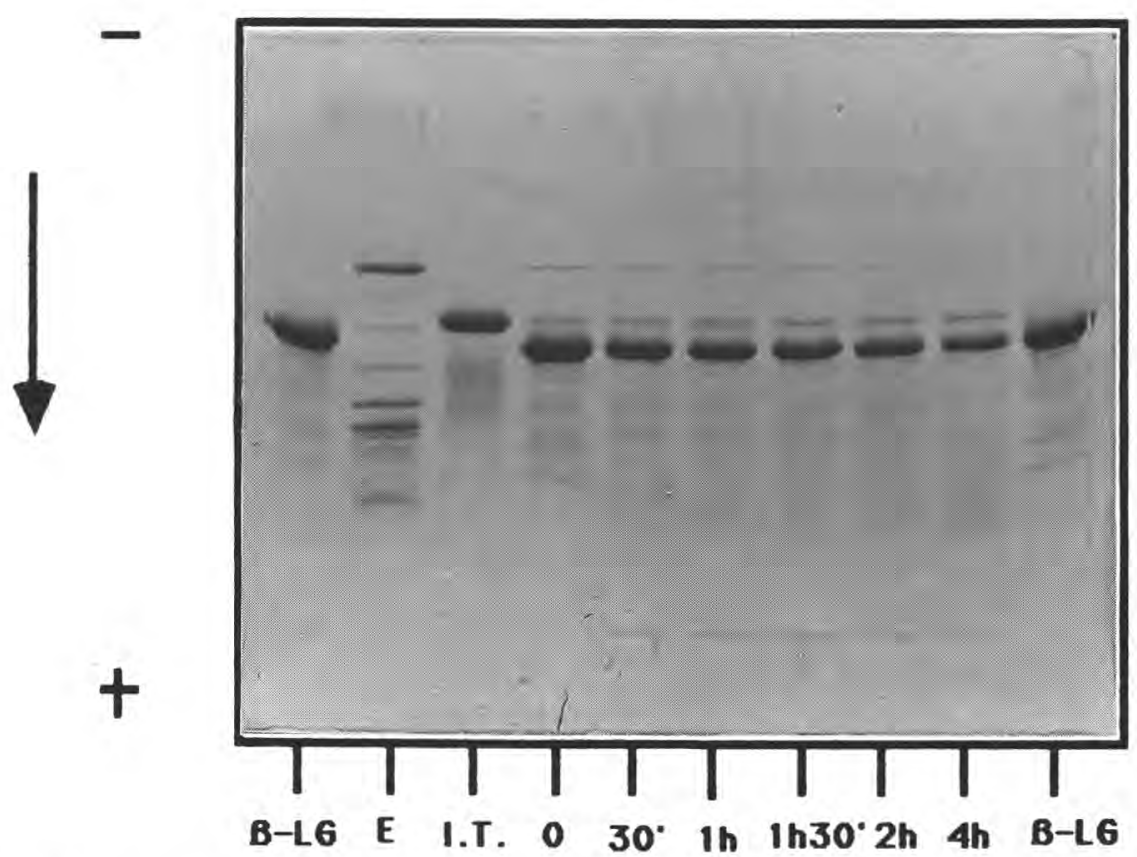

Fig 2. Electrophorèse en gel de polyacrylamide à $15 \%$ d'acrylamide en milieu SDS des hydrolysats trypsiques de la $\beta$-lactoglobuline obtenus dans les conditions de la figure 1 avec $E / S=1 / 25$ ( $\beta$-LG: $\beta$ lactoglobuline; $\mathrm{E}$ : témoin trypsine; IT: témoin inhibiteur trypsique; $0-4 \mathrm{~h}$ : hydrolysats aux temps 0,30 $\min , 1 \mathrm{~h}, 1 \mathrm{~h} 30 \mathrm{~min}, 2 \mathrm{~h}$ et $4 \mathrm{~h}$ ).

Analysis by SDS-PAGE at $15 \%$ acrylamide of the reaction mixture obtained as described in figure 1 with $E / S=1 / 25$ ( $\beta$-LG: $\beta$-lactoglobulin; $E$ : control trypsin; IT: control soybean inhibitor; 0-4 h: hydrolysis times $0,30 \mathrm{~min}, 1 \mathrm{~h}, 1 \mathrm{~h} 30 \mathrm{~min}, 2 \mathrm{~h}$ and $4 \mathrm{~h}$ ).

évidence par l'analyse électrophorétique. Ceci traduit non seulement la dénaturation partielle et la déstabilisation de structure mais bien des changements importants de la structure protéique, ce qui est une observation intéressante à souligner. La coupure de certaines liaisons peptidiques fragiles se produit au cours du traitement de la $\beta$-lactoglobuline et un mélange de polypeptides de différentes tailles est obtenu.

L'augmentation de la vitesse d'hydrolyse par la trypsine doit sans doute être attribuée à une hydrolyse facilitée de ces produits de dégradation. Aussi, les processus mis en jeu dans la dénaturation thermi- que en milieu acide dilué ne peuvent être reliés aux changements conformationnels consécutifs à une thermodénaturation à $\mathrm{pH}$ neutre étudiée par Mohan Reddy et al (1988) qui augmentent la susceptibilité à l'hydrolyse.

\section{$\beta$-lactoglobuline réduite}

La solubilité de la $\beta$-lactoglobuline réduite est très limitée : 95\% d'insoluble (tableau I). Cette baisse de solubilité suite à une réduction des ponts disulfures a été observée par Kella et al (1989) pour les pro- 


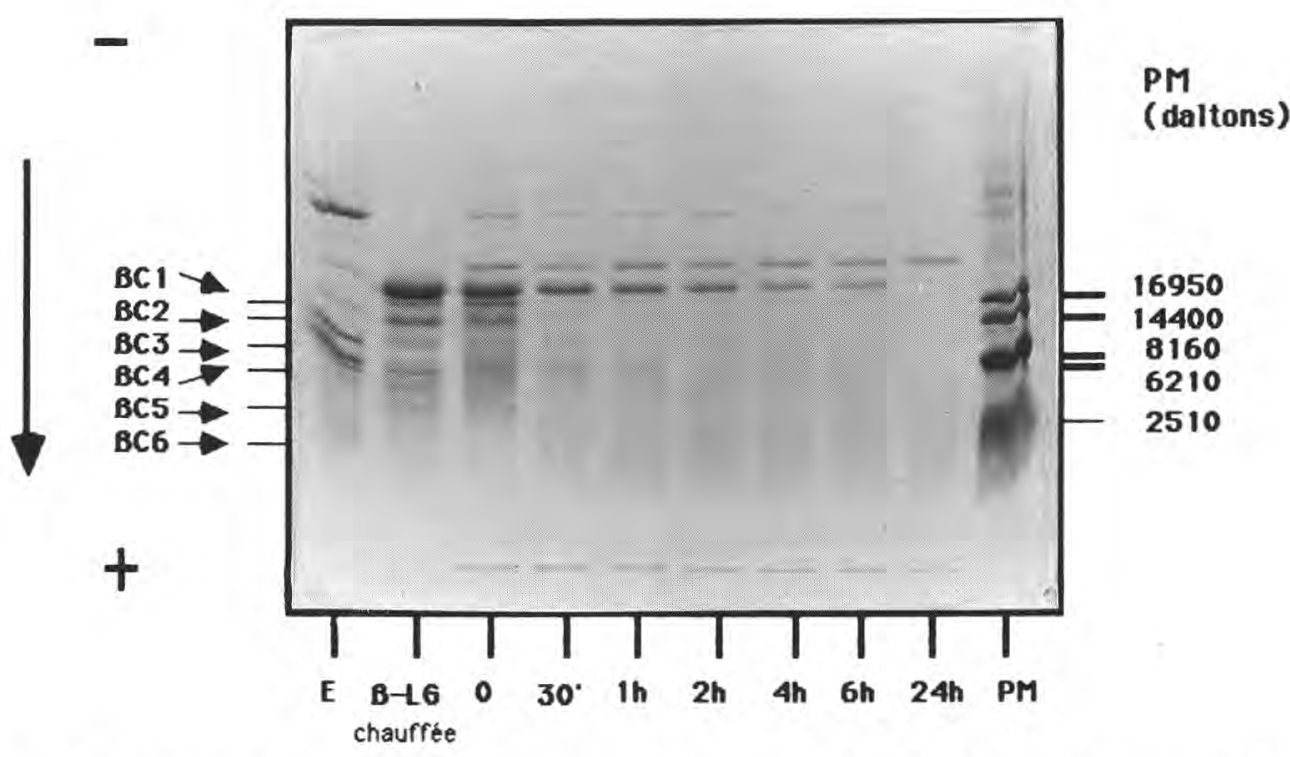

Fig 3. Electrophorèse en gel de polyacrylamide à $15 \%$ en milieu SDS des hydrolysats trypsiques de la $\beta$-lactoglobuline chauffée à $90^{\circ} \mathrm{C}$ en milieu acide $\mathrm{HCl} 0,02 \mathrm{M}$ obtenus dans les conditions : concentration en $\beta$-lactoglobuline: $0,5 \%$; rapports enzyme/substrat $(p / p): 1 / 25$; tampon phosphate de sodium $0,2 \mathrm{M} \mathrm{pH} 7,5$; température : $37{ }^{\circ} \mathrm{C}$ ( $\beta$-LG: $\beta$-lactoglobuline; $\mathrm{E}$; témoin trypsine; PM: marqueurs de poids moléculaire; $0-24 \mathrm{~h}$ : hydrolysats aux temps $0,30 \mathrm{~min}, 1 \mathrm{~h}, 2 \mathrm{~h}, 4 \mathrm{~h}, 5 \mathrm{~h}$ et $24 \mathrm{~h} ; \beta \mathrm{C} 1-\beta \mathrm{C}$ : fragments peptidiques).

Analysis by SDS-PAGE at $15 \%$ acrylamide of heated $\beta$-lactoglobulin in acid media $10.5 \%$ solution in $0.02 \mathrm{M} \mathrm{HCl}$ heated at $90{ }^{\circ} \mathrm{C}, 1 \mathrm{~h}$ ) and of treated $\beta$-lactoglobulin hydrolysed by trypsin. Treated $\beta$ lactoglobulin concentration: $0.5 \%$; $E / S$ ratio $(w / w)$ : $1 / 25$; Na phosphate buffer $0.2 \mathrm{M} \mathrm{pH} 7.5$; temperature : $37^{\circ} \mathrm{C}$ ( $\beta$-LG; treated $\beta$-lactoglobulin; E: control trypsin; PM: molecular weight markers; 0-24 h: hydrolysis times $0,30 \mathrm{~min}, 1 \mathrm{~h}, 2 \mathrm{~h}, 4 \mathrm{~h}, 5 \mathrm{~h}$ and $24 \mathrm{~h} ; \beta C 1-\beta C 6$ : peptides).

téines de lactosérum, Song et Damodaran (1987) pour la sérumalbumine bovine et Kim et Kinsella (1986) pour la glycinine de soja. Cela traduit le dépliement de la molécule et l'exposition des groupements hydrophobes préalablement enfouis à l'intérieur de la structure, favorisant les interactions protéine/protéine.

Avec la trypsine utilisée dans un rapport E/S de $1 / 25$, un degré d'hydrolyse de $8 \%$ est atteint après $5 \mathrm{~min}$ et de $12 \%$ après $120 \mathrm{~min}$, soit une vitesse initiale estimée à 50 unités $\mathrm{DH} / \mathrm{min}$ comparativement à 0,4 unité $\mathrm{DH} / \mathrm{min}$ pour le témoin de $\beta$ - lactoglobuline native. Le rapport E/S de 1/ 250 conduit à une hydrolyse plus progressive de la $\beta$-lactoglobuline réduite $(0,8$ unité $\mathrm{DH} / \mathrm{min}$ ) et un degré d'hydrolyse de $8 \%$ est atteint après $30 \mathrm{~min}$.

La déstabilisation des structures secondaire et tertiaire de la $\beta$-lactoglobuline facilite l'accessibilité de la trypsine aux liaisons peptidiques. Cela peut être rapproché de l'augmentation de la vitesse d'hydrolyse observée par Church et al (1982) après modification de la structure de la $\beta$ lactoglobuline en milieu urée 6 et $8 \mathrm{M}$ lors de l'hydrolyse par la pronase de Strepto- 
myces griseus. Otani (1981) signale l'influence positive de la réduction des ponts disulfures de la $\beta$-lactoglobuline sur l'augmentation de la vitesse d'hydrolyse pepsique, trypsique et chymotrypsique. Plus récemment, Mohan Reddy et al (1988) ont relié la coupure des liaisons disulfures de la $\beta$-lactoglobuline à des changements conformationnels très importants appréciés par une altération du spectre d'émission par fluorescence et une décroissance de polarité de surface qui reflète l'exposition de groupements hydrophobes; suite à la perte d'intégrité structurale, la résistance à la digestion pepsique ou chymotrypsique décroît.

Les profils électrophorétiques de ces hydrolysats (fig 4) permettent d'identifier les peptides issus de l'hydrolyse trypsique : $\beta R 1$ à $17600, \beta R 2$ à $15000, \beta R 3$ à 13200 et $\beta R 4$ à 5000 . Pour le rapport E/S $=1 / 25$, ils apparaissent dès la mise en contact de l'enzyme et du substrat et après 5 min ces bandes principales et la $\beta$ lactoglobuline ne sont plus décelables. Pour le rapport $E / S=1 / 250$, la mise en contact de l'enzyme et du substrat provoque l'apparition des mêmes produits, ceux de poids moléculaires plus élevés étant les plus importants (intensité : $\beta$-lactoglobuline $>\beta R 1>\beta R 2>\beta R 3>\beta R 4)$. Ces produits sont à leur tour hydrolysés, $\beta R 2$ et $\beta R 4$ restant identifiables jusqu'à 30 min.

L'analyse électrophorétique nous permet ici de mettre en évidence un processus d'hydrolyse trypsique de la $\beta$ lactoglobuline tout à fait différent après la perte de son intégrité structurale : des peptides intermédiaires de taille importante (5000 à 17 600) apparaissent dans le milieu réactionnel et traduisent une attaque préférentielle par la trypsine de certaines des liaisons "sensibles" (au niveau des ré- sidus Arg et Lys). Cela est certainement dû à un environnement favorisant des interactions électrostatiques avec le site catalytique de la trypsine ou des interactions hydrophobes permettant l'association enzyme/substrat (Fontana et al, 1986; Novotny et Bruccoleri, 1987).

\section{CONCLUSION}

Nos résultats montrent l'importance des traitements de dénaturation de la $\beta$ lactoglobuline sur la nature des produits obtenus avant et après l'hydrolyse trypsique. L'hydrolyse trypsique du substrat dans l'état natif conduit à l'apparition de peptides de petites tailles, non identifiables par l'analyse électrophorétique employée.

Le traitement thermique en milieu acide très dilué entraîne l'apparition d'un mélange de polypeptides de différentes tailles identifiés par analyse électrophorétique en milieu dissociant. Ces observations sur la nature des produits obtenus apportent des éléments nouveaux dans la compréhension des mécanismes de dénaturation protéique. Les propriétés de ces produits font l'objet d'une étude complémentaire, et en particulier les relations entre les propriétés émulsifiantes et le degré de dénaturation appliqué (couples temps-température) (lung, 1988).

La trypsine hydrolyse la $\beta$-lactoglobuline chauffée en milieu acide ou réduite par des mécanismes différents. Ainsi, la coupure des ponts disulfure et la perte des structures tertiaire et secondaire qui l'accompagne permet d'identifier des peptides dont la taille est comprise entre 5000 et 17600 ; ces peptides n'ont pas été décelés lors de l'attaque trypsique de la $\beta$ lactoglobuline chauffée en milieu acide. 

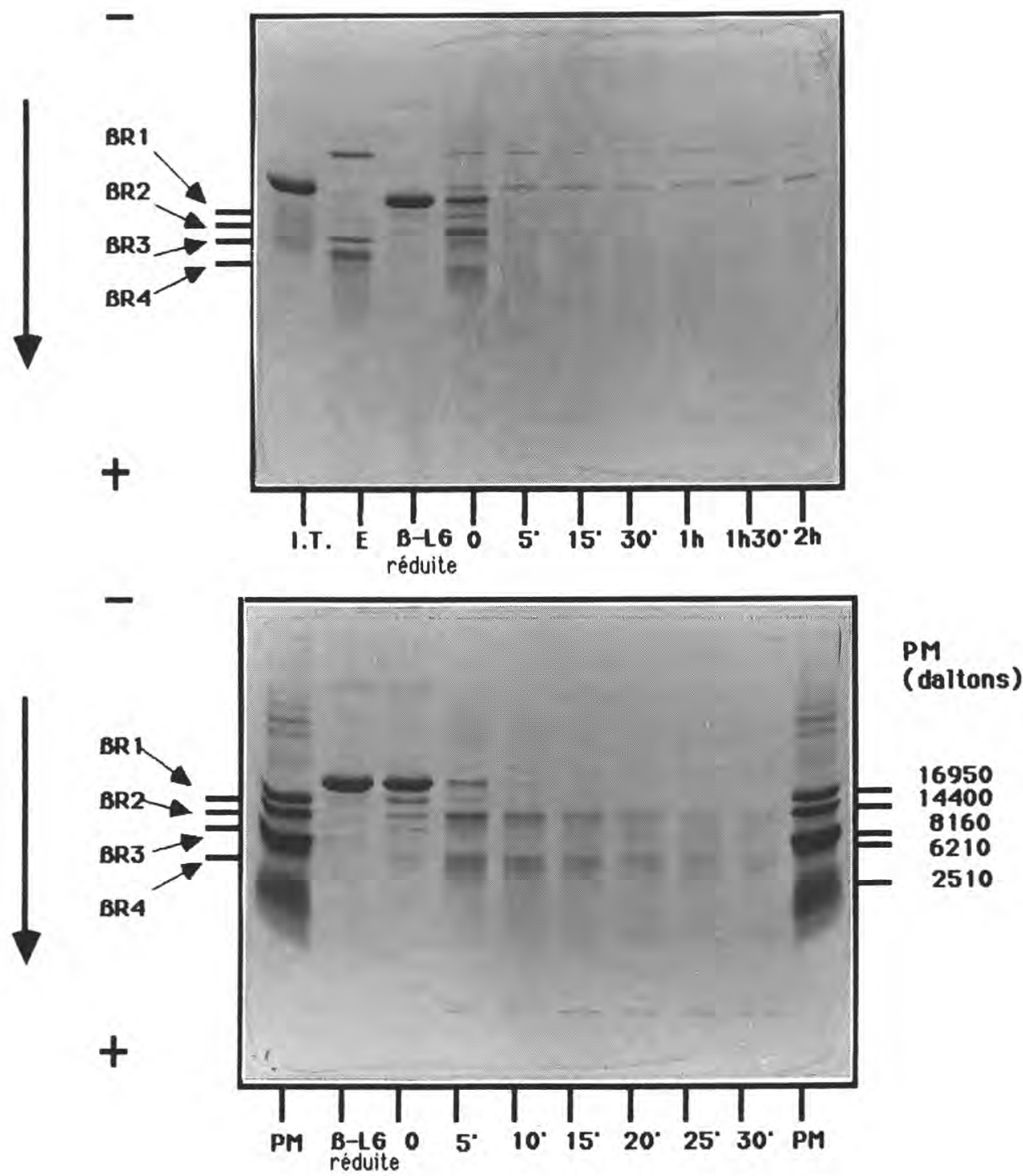

Fig 4. Electrophorèse en gel de polyacrylamide à $15 \%$ en milieu SDS des hydrolysats trypsiques de la $\beta$-lactoglobuline réduite obtenus dans les conditions : a) concentration en $\beta$-lactoglobuline: $1 \%$; rapport enzyme/substrat $(\mathrm{p} / \mathrm{p}): 1 / 25$; tampon phosphate de sodium $0,2 \mathrm{M} \mathrm{pH} 7,5$; température : $37^{\circ} \mathrm{C}$. b) concentration en $\beta$-lactoglobuline: $0,5 \%$; rapport enzyme/substrat $(p / p): 1 / 250$; tampon phosphate de sodium $0,2 \mathrm{M} \mathrm{pH} 7,5$; température : $37^{\circ} \mathrm{C}$ ( $\beta$-LG: $\beta$-lactoglobuline; $E$ : témoin trypsine; IT: témoin inhibiteur trypsique; PM: marqueurs de poids moléculaire; $0-2 \mathrm{~h}$ : hydrolysats aux temps $0,5 \mathrm{~min}, 15 \mathrm{~min}$, $30 \mathrm{~min}, 1 \mathrm{~h}$ et $2 \mathrm{~h} ; 0-30 \mathrm{~min}$ : hydrolysats aux temps $0,5,10,15,20,25$ et $30 \mathrm{~min} ; \beta R 1-\beta R 4$ : fragments peptidiques).

Analysis by SDS-PAGE at $15 \%$ acrylamide of reduced $\beta$-lactoglobulin and reduced $\beta$-lactoglobulin hydrolysed by trypsin. a) reduced $\beta$-lactoglobulin in concentration: 1\%; E/S ratio (w/w); 1/25; Na phos-

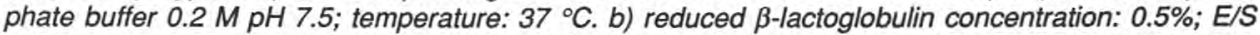
ratio (w/w): 1/250; Na phosphate buffer $0.2 \mathrm{M} \mathrm{pH}$ 7.5: temperature : $37{ }^{\circ} \mathrm{C}$ ( $\beta$-LG: reduced $\beta$ lactoglobulin; E: control trypsin; IT: control soybean inhibitor; PM: molecular weight markers: 0-2 h: hydrolysis times $0,5 \mathrm{~min}, 15 \mathrm{~min}, 30 \mathrm{~min}, 1 \mathrm{~h}$ and $2 \mathrm{~h} ; 0-30 \mathrm{~min}$; hydrolysis time 0, 5, 10, 15, 20, 25 and 30 min; $\beta R 1-\beta R 4$ : peptides). 


\section{RÉFÉRENCES}

Cheftel JC, Cuq JL, Lorient D (1985) Protéines alimentaires, propriétés fonctionnelles, valeur nutritionnelle, modifications chimiques. Tech et Doc Lavoisier, Paris

Church FC, Catignani GL, Swaisgood HE (1982) Use of immobilized Streptomyces griseus proteases (pronase) as a probe of structural transitions of lysozyme, $\beta$ lactoglobulin and casein. Enzyme Microb Technol 4, 317-321

Church FC, Porter DH, Catignani GL, Swaisgood HE (1985) An o-phtaldehyde spectrophotometric assay for proteinases. Anal Biochem 146, 343-348

Dalgalarrondo $M$, Chobert JM, Dufour E, Bertrand-Harb C, Dumont JP, Haertlé T (1990) Characterization of bovine $\beta$-lactoglobulin B tryptic peptides by reversed-phase high performance liquid chromatography. Milchwissenschaft 45, 212-216

Fontana A, Fassina G, Vita C, Dalzoppo D, Zamai M, Rambonin M (1986) Correlation between sites of limited proteolysis and segmental mobility in thermolysin. Biochemistry $25,1847-1851$

Harwalkar VR (1980) Measurement of thermal denaturation of $\beta$-lactoglobulin at $\mathrm{pH} 2.5 . \mathrm{J}$ Dairy Sci 63, 1043-1051

lung C (1988) Les propriétés fonctionnelles des protéines de lactosérum. Étude modélisée avec la $\beta$-lactoglobuline. Thèse Univ, Nancy I

lung C, Pâquet $D$, Linden $G$ (1987) Isolement de la $\beta$-lactoglobuline. Mise au point d'une méthode préparative. Sci Aliments 7, 167-176

Jakobsson I, Lindberg T, Benediktsson B, Hanson BG (1985) Dietary bovine $\beta$-lactoglobulin is transferrd to human milk. Acta Pediatr Scan 74, 341-345

Jost R (1988) Physico-chemical treatment of food allergens: application to cow's milk protein. Food Allergy 17, 187-197

Kella NKD, Yang ST, Kinsella JE (1989) Effect of disulfide bond cleavage on structural and interfacial properties of whey proteins. $J$ Agric Food Chem 37, 1203-1210

Kim SH, Kinsella JE (1986) Effects of reduction with dithiothreitol on some molecular proper- ties of soy glycinin. J Agric Food Chem 34, 623-627

Konisberg W (1972) Reduction of disulfide bonds in proteins with dithiothreitol. In: Methods in Enzymology. XXV. Enzyme structure. Part B (CHW Hirs, SN Timasheff, eds) Academic Press, New York, London, 185186

Laemmli UK, Favre M (1973) Maturation of the head of bacteriophage T4. I. DNA packaging events. J Mol Biol 80, 575-599

Lowry OH, Rosebrough NJ, Farr AL, Randall RJ (1951) Protein measurement with the Folin phenol reagent. J Biol Chem 193, 265-275

Miranda G, Pélissier JP (1983) Kinetic studies of in vivo digestion of bovine unheated skimmilk proteins in the rat stomach. I Dairy Res 50, 27-36

Mohan Reddy IM, Kella NKD, Kinsella JE (1988) Structural and conformational basis of the resistance of $\beta$-lactoglobulin to peptic and chymotryptic digestion. J Agric Food Chem 36, 737-741

Novotny J, Bruccoleri RE (1987) Correlation among sites of limited proteolysis, enzyme accessibility and segmental mobility. FEBS Lett 211, 185-189

Otani H (1981) Susceptibility of Scarboxymethylated $\beta$-lactoglobulin to peptic, tryptic and chymotryptic digestions. Jpn Zootech Sci 52, 689-691

Papiz MZ, Sawyer L, Eliopoulos EE, North ACT, Findlay JBC, Sivaprasadarao R, Jones TA, Newcomer ME, Kraulis PJ (1986) The structure of $\beta$-lactoglobulin and its similarity to plasma retinol-binding protein. Nature 324, 383-385

Sawyer L, Papiz MZ, North ACT, Eliopoulos EE (1985) Structure and function of bovine $\beta$ lactoglobulin. Biochem Soc Trans 13, 265266

Shimizu M, Saito M, Yamauchi K (1985) Emulsifying and structural properties of $\beta$ lactoglobulin at differents $\mathrm{pHs}$. Agric Biol Chem 49, 189-194

Song KB, Damodaran S (1987) Structurefunction relationship of proteins: adsorption of structural intermediates of bovine serum albumin at the air-water interface. $J$ Agric Food Chem 35, 236-241 
Waniska RD, Kinsella JE (1985) Surface properties of $\beta$-lactoglobulin: adsorption and rearrangement during film formation. J Agric Food Chem 33, 1143-1148
Yvon M, Van Hille I, Pélissier JP, Guilloteau P, Toullec $R$ (1984) In vivo milk digestion in the calf abomasum II. Milk and whey proteolysis. Reprod Nutr Dev 24, 835-843 